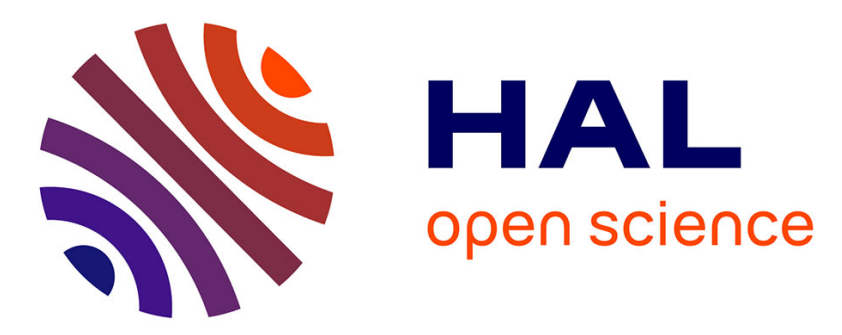

\title{
Triggering and acceleration of xylitol crystallization by seeding and shearing: Rheo-optical and rheological investigation
}

Mónica Delgado, Miguel Navarro, Ana Lázaro, Séverine A.E. Boyer, Edith Peuvrel-Disdier

\section{To cite this version:}

Mónica Delgado, Miguel Navarro, Ana Lázaro, Séverine A.E. Boyer, Edith Peuvrel-Disdier. Triggering and acceleration of xylitol crystallization by seeding and shearing: Rheo-optical and rheological investigation. Solar Energy Materials and Solar Cells, 2021, 220, pp.110840. 10.1016/j.solmat.2020.110840 . hal-03052826

\section{HAL Id: hal-03052826 \\ https://hal.science/hal-03052826}

Submitted on 2 Jan 2021

HAL is a multi-disciplinary open access archive for the deposit and dissemination of scientific research documents, whether they are published or not. The documents may come from teaching and research institutions in France or abroad, or from public or private research centers.
L'archive ouverte pluridisciplinaire HAL, est destinée au dépôt et à la diffusion de documents scientifiques de niveau recherche, publiés ou non, émanant des établissements d'enseignement et de recherche français ou étrangers, des laboratoires publics ou privés. 
1 Title: Triggering and acceleration of xylitol crystallization by seeding and shearing: Rheo-

2 optical and rheological investigation

3 Mónica Delgado ${ }^{\mathrm{a}, \mathrm{b}^{*}}$, Miguel Navarro ${ }^{\mathrm{b}}$, Ana Lázaro ${ }^{\mathrm{b}}$, Séverine A.E. Boyer ${ }^{\mathrm{c}}$, Edith Peuvrel-

4 Disdier $^{\mathrm{c}}$

5 a Centro Universitario de la Defensa, Academia General Militar, Ctra. Huesca S/N, 50090

6 Zaragoza, Spain

7 bAragón Institute for Engineering Research (I3A), Thermal Engineering and Energy Systems

8 Group, University of Zaragoza, Agustín Betancourt Building, C/María de Luna 3, 50018,

9 Zaragoza, Spain

10 'MINES Paris, PSL Research University, CEMEF-Centre de Mise en Forme des Matériaux,

11 UMR CNRS 7635, CS 10207, 06904 Sophia-Antipolis, France

$12 *$ Corresponding author e-mail: $\underline{\text { monica@unizar.es }}$

\section{Abstract}

The present study investigates the triggering and acceleration of xylitol crystallization for its use as a short-term TES. The combined effect of seeding and the action of shear on xylitol crystallization at a supercooled temperature has been investigated by direct observations under shear (rheo-optical approach) and by rheometry. The initial seed is an agglomerate of crystals of xylitol. In addition to the more classical erosion and rupture mechanisms leading to the dispersion of crystals, shear has shown to induce the detachment of crystalline dust adhered to the initial seed into the supercooled xylitol. These crystal fragments then serve as sites for secondary nucleation and subsequent crystallization. Rheo-optical observations have allowed the determination of shear conditions to control the size of crystals by dispersion. A second part has been dedicated to the effect of shear on crystallization of xylitol by seeding by means of rheology. The measurements, although not reproducible, clearly show that seeding in presence of shear (for the investigated conditions) is very efficient to trigger and accelerate the crystallization of xylitol. Post-mortem DSC and XRPD analyses of the final crystallized seeded and sheared samples have not shown any effect on the xylitol crystallinity degree.

Keywords: Phase Change Materials, Sugar alcohols, Crystallization, Secondary nucleation, Shear

\section{Introduction}

The availability of renewable energy sources, more favourable thermal sources or waste heat sources is generally of an intermittent nature and often does not satisfy demand. Thus, thermal energy storage (TES) is required to increase the exploitation of these resources seeking a more sustainable energy model. Latent TES by means of the solid-liquid phase change of materials shows a high energy storage density. Phase change materials (PCM) can store up to 7 times more energy per volume unit than by sensible heat, such as water in a temperature range of $15^{\circ} \mathrm{C}$. These materials can be classified by their nature as inorganic PCMs, such as salts and hydrated salts, or organic PCMs, such as paraffins, fatty acids and sugar alcohols. Sugar 
alcohols are low-digestible carbohydrates obtained by substituting an aldehyde group with a hydroxyl group. Polyols are naturally present in small quantities in fruits as well as in certain kinds of vegetables or mushrooms [1]. They show a high energy storage density, more than twice that of paraffins, the most commonly used PCM. Furthermore, they are of natural origin, non-toxic, non-flammable, non-corrosive and cheap. Nevertheless, they have received little attention due to the fact that most polyols have a melting temperature between 90 and $200^{\circ} \mathrm{C}$ and are therefore excluded from heating and domestic hot water (DHW) applications. In recent years, several researchers have developed new mixtures of sugar alcohols so as to be able to use these polyols in applications below $100^{\circ} \mathrm{C}$ [2-4].

Another characteristic of sugar alcohols is their supercooling. Supercooling is usually considered as a drawback, since to be able to discharge the energy stored, the temperature should fall below its crystallization temperature. However, supercooling can be advantageous for long-term or seasonal energy storage. In particular, xylitol has stable supercooling properties, but its difficult nucleation triggering hinders its use as a PCM and its low crystallization rate means that its thermal energy discharge is inadequate for TES applications [5]. Several works have studied the triggering and acceleration of the crystallization process [6]. The problems of the nucleation and low crystallization rate also apply to xylitol, the sugar alcohol studied here.

Xylitol is a 5-carbon polyol produced from D-xylose. It was discovered in 1891 and since the 1960s has been used as a sweetener. The main substrate for its production is xylan, which is usually obtained from birch trees and other hardwood. Similar to other sugar alcohols, xylitol is produced by metal catalysed hydrogenation of a corresponding sugar, i.e., D-xylose [1]. Some alternative biotechnological processes are currently being studied in order to reduce production costs [7].

There are few studies in the literature concerning the nucleation and crystallization of xylitol for its use as a PCM. Seppäla et al. [5] added some additives to xylitol for the purposes of decreasing the surface tension between the solid-liquid phase in the crystallization front and of decreasing the viscosity. The only additives that triggered the crystallization with a supercooling of $70^{\circ} \mathrm{C}$ were ethanol and acetone. The mixtures formed by the rest of the additives required some xylitol crystals to be dropped onto the top surface of the melted mixture in order to initialize the crystallization of the top layer. All the additives increased the crystallization rate. The highest crystallization rate was observed with a 5\% acetone, ethanol and methanol concentration, being 10 times higher than that of pure xylitol. In spite of this improvement, the crystallization rate was insufficient from the point of view of common TES applications. Godin et al. [8] also analysed different ways of triggering xylitol crystallization. Among the different techniques proposed, local cooling did not achieve nucleation due to the high activation energy required for the diffusion of atoms and realignment in the solid-liquid interphase. Seeding was also considered, but its effect was very local and the crystallization rate too low for the material to be considered feasible for TES applications. A similar result was observed with high power ultrasounds. Finally, the authors proposed the bubbling technique to trigger the supercooled xylitol crystallization and its acceleration. The crystallization occurred at the surface of the 
small drops generated at the liquid surface, when the primitive bubbles exploded into daughter bubbles. Duquesne et al. [9] observed that mechanical agitation of a $400 \mathrm{ml}$ xylitol sample contained in a glass beaker started to nucleate in only $5 \mathrm{~s}$ of mixing throughout the agitated region with an undercooling of $45^{\circ} \mathrm{C}$.

The addition of nanoscale fillers was studied by Salvan and Suresh [10-11] on some sugar alcohols such as d-mannitol -but not xylitol- with the aim of improving the nucleation and crystal growth, while improving the thermal conductivity. In their first study [10], they added multi-walled carbon nanotubes in d-mannitol samples. Supercooling was hardly decreased, making its application unfeasible as a short term TES. The crystallization time was reduced by $17 \%$ in presence of nanoparticles. In their second study [11], they added copper oxide nanoparticles. Although the supercooling problem was still present, they managed to reduce the crystallization time by $67 \%$. The microencapsulation of d-mannitol in a silica shell was also adopted as a potential solution to improve its thermophysical properties [12]. The authors succeeded in reducing the supercooling from 44 to $11^{\circ} \mathrm{C}$, where silica shell acted as nucleation site. Althougth data about the crystallization rate is not directly provided, they show the results of a cooling experiment, being the time taken to be cooled from 160 to $40^{\circ} \mathrm{C}$ the same both for the encapsulated and non-encapsulated samples.

Crystallization proceeds by two consecutive events: 1) nucleation, the generation of new crystals (nuclei), followed by 2) growth, where the nuclei become larger crystals. The formation of the new nuclei can occur via primary or secondary mechanisms. Secondary nucleation uses crystals as a site and source for the formation of nuclei and plays an important role in determining the product size distribution [13].

The mechanism whereby secondary nuclei originate from the crystal can occur by: 1) Initial breeding, where fine crystalline dust attached to the surface of larger crystals is dislodged, becoming nuclei. This can be minimized by washing the seed crystals [14]. The number of fines on a seed crystal surface is finite and therefore initial breeding cannot be a sustained source of nucleation.

2) Dendritic or needle breeding, which occurs over the crystal surface. The needles then detach from the main crystal due to mechanical forces and grow as independent crystals [15]. However, this mechanism is unlikely to happen due to the low crystallization rate.

3) Attrition or erosion, which refers to the removal of fragments from the parent crystal due to collisions with each other or other objects, or by the flow of the suspending fluid, due to hydrodynamic stresses created by the flow of the fluid around the particle. The generated fragments act as nuclei. The damaged parent crystals, as well as macro attrition fragments (secondary nuclei), are observable immediately after the attrition event because of their comparatively larger size. This contrasts with contact/shear nucleation, where no apparent damage to the crystal occurs and there is a time delay in the appearance of the secondary nuclei, with nuclei requiring time to grow to a detectable size [16].

The present work examines in depth xylitol crystallization by shearing, a technique not to date reported in the literature for sugar alcohols. In this study, xylitol nucleation was triggered by the 
introduction of xylitol crystal seeds. Seeding alone was not considered because its effect is too local and the crystal growth is too slow. In fact, industrial crystallizers use seeding in combination with stirring to create and maintain a uniform dispersion of the seeds in the bulk and to improve the mass transfer rate between the solid and liquid phase. Shearing affects nucleation, the orientation of the crystals formed, their structure and size, and the polymorph transitions. In addition, shearing can promote crystal aggregation or, if the shear rate is very high, it can promote the breaking of these aggregates [17-18]. Specifically, this research aims at the triggering and acceleration of xylitol crystallization by means of self-seeding under temperature and shear conditions with the objective of using xylitol as a short-term TES. It is proposed to monitor the secondary nucleation phenomenon and subsequent crystallization when xylitol seeds are added to the supercooled xylitol, using a microscope coupled with a transparent counter-rotating shear cell and a rheometer. The originality of the research lies in this rheooptical approach, which allows direct observation in-situ of the xylitol during shear. Furthermore, the effect of shear on crystallization has been investigated by rheology. Micrographs of the sheared samples have been taken showing differences in the crystal sizes. To analyse possible changes in the crystallinity of the thermo-sheared samples, Differential Scanning Calorimetry (DSC) and X-ray powder diffraction (XRPD) measurements have been performed. The results of the present research could be the starting point for the design of xylitol stirred tanks to be used for short-term TES. While in the rheology experiments, the shear rate is controlled and modified, in a stirred TES tank, what would be modified is the rotation speed of the impeller.

\section{Materials and methods}

\subsection{Materials}

Xylitol $\left(\mathrm{C}_{5} \mathrm{H}_{12} \mathrm{O}_{5}\right.$, CAS number 87-99-0) of $99 \%$ purity from Sigma Aldrich was used. Karl Fischer titration measurements were performed to ensure negligible water content. Moisture sorption isotherms showed that at room temperature and relative humidities below $75 \%$, the mass variation was lower than $0.1 \%$. It can be stated that moisture was not adsorbed during the preparation of samples. Two crystalline forms of xylitol were reported in the early literature: a metastable, hygroscopic monoclinic form melting at $61^{\circ} \mathrm{C}$ and a stable orthorhombic form melting at $94^{\circ} \mathrm{C}[19]$.

Xylitol seeds with a diameter range between 315 and $400 \mu \mathrm{m}$ were obtained from the xylitol chunks by sieving as directly received by Sigma Aldrich. The crystal form of the seeds according to DSC and XRPD experiments was orthorhombic.

\subsection{Methods}

\subsubsection{Rheo-optical shear experiments}

A counter-rotating shear cell [20] was used to perform the rheo-optical shear experiments. The shear cell consists of two transparent parallel glass disks, independently driven by two motors in order to rotate in opposite directions. The sample is introduced in between the two glass plates. The counter-rotating option is very useful to observe continuously the behaviour of an object 
suspended in a fluid medium while being submitted to a shear. The relative velocities of the two plates can be adjusted so that the velocity of the suspended particle can be set to zero in the reference framework of the laboratory. This shear cell is mounted on an optical microscope (Metallux 3 from Leica) equipped with a CCD Camera. Figure 1 shows the lower and upper plates of the shear cell. The temperature is controlled by heating elements placed under the lower plate, and also by an oven surrounding the sheared zone, enclosed by two semi-discs of quartz to avoid heat losses and permit optical observations [21]. The temperature was checked with benzoic acid. A deviation of $1.5^{\circ} \mathrm{C}$ between the melting temperature and the certified temperature was observed at the observation window for an applied shear rate of $10 \mathrm{~s}^{-1}$.

Xylitol crystal seeds were added prior to the shear. All experiments were conducted in isotherm conditions at the supercooled temperature of $90^{\circ} \mathrm{C}$. This set temperature was selected keeping in mind the application of xylitol for a short-term TES. Although the melting temperature is $94^{\circ} \mathrm{C}$ [19], it was decided to test at a slightly lower temperature to prevent possible overheating and subsequent melting during the temperature control of the device.

The sample was loaded in three steps. First, half of the molten xylitol (pre-heated in a beaker placed in a temperature-controlled bath at the supercooled temperature) was loaded onto the lower plate, whose temperature was controlled. Next, three seeds were placed in the supercooled xylitol. Finally, the other half of the molten xylitol was loaded. Once loaded, the gap was set at $600 \mu \mathrm{m}$ and shear history could be applied. The gap was selected to avoid any friction of the seed with the glass walls.

The first step in these experiments was to find one of the seeds added to the supercooled xylitol to trigger the crystallization. This was performed by rotating both plates at the same speed (low rotation speed). Once the seed was found, a low shear rate was applied in counter-rotating mode. Velocities were chosen so that the position of the seed remains fixed relatively to the laboratory framework, while submitted to a shear flow. The shear induces the slow rotation of the seed, allowing its observation in all directions. Then the shear rate was progressively increased to observe its effect on the initial seed. The shear rate was adjusted manually. Shear rates up to $100 \mathrm{~s}^{-1}$ were investigated. If the shear rate was changed too abruptly, the seed got lost, and another one was searched for observation.

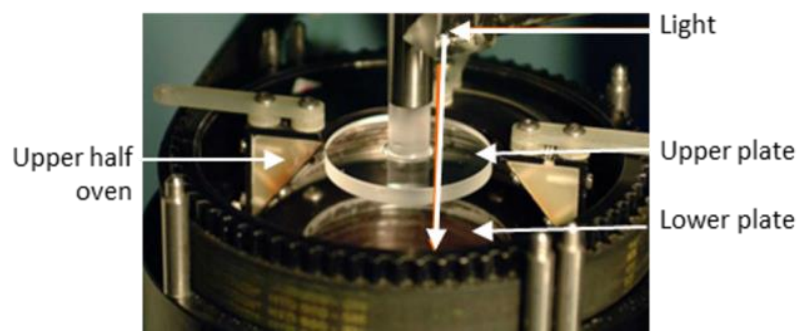

Figure 1. Transparent lower and upper plates of the counter-rotating shear cell [21]

\subsubsection{Rheological measurements}

The influence of shear on crystallization at different supercooling and shear rates was investigated with a controlled stress rheometer from TA Instruments (AR-G2 model). For these 
experiments, a Peltier plate was used as a temperature controller and a plate with a diameter of $40 \mathrm{~mm}$ as the geometry. Although the parallel plate geometry has the drawback of a linear shear rate variation along the radius, it was chosen because the cone-and-plate $(1 \% / 40 \mathrm{~mm})$ geometry available in the laboratory, with a gap at the edge of $380 \mu \mathrm{m}$, prevented the seed addition. The seed diameter was too big in comparison to the distance between the cone and the plate. Previous experiments performed with a standard oil in the parallel plate configuration showed a deviation from the certified viscosity value of $8 \%$ at $80^{\circ} \mathrm{C}$ as a result of the temperature gradient within the sample with a gap of $645 \mu \mathrm{m}$ [22]. Lower gaps would reduce this temperature gradient. However, due to the seed size, a gap of $600 \mu \mathrm{m}$ was finally chosen to perform the shear experiments, bigger than the seed size range to limit friction of the seeds on the plates during the measurements. The shear rate values executed were 5, 10 and $15 \mathrm{~s}^{-1}$, and they correspond to the shear rate at the edge of the geometry, i.e. the maximum shear rate.

From the point of view of the application where the TES could be integrated, the shear rate range will depend on the required charge thermal power. In a previous study carried out by some of the authors of the present article, a tank containing a paraffin emulsion for heating applications was also stirred to improve its thermal response. In that case, a rotation speed of $230 \mathrm{rpm}$ was enough to increase approximately five times the overall heat transfer coefficient [23]. The impeller of the stirred tank was a trilight impeller. This rotation condition is estimated to correspond to an average rate of $13 \mathrm{~s}^{-1}$ (average shear rate calculated through correlations found in literature [24], which is within the shear rate range of the rheological experiments performed.

The protocol to load the sample and introduce the crystal seeds was similar to that used for the rheo-optical observations, but adding only one seed. Once the whole sample was loaded, the viscosity and normal force were measured over time at a predefined shear rate and supercooling temperature. In fact, two protocols were tested, a direct application of the shear rate of measurement, or the application of a pre-shear rate prior to the predefined shear rate. The preshear rate was chosen above the determined critical shear rate by rheo-optical observations in order to break the initial seed. $50 \mathrm{~s}^{-1}$ for $1 \mathrm{~min}$ was applied as pre-shear. Once the sample was crystallized, some crystals were taken for subsequent analysis by DSC and XRPD.

\subsubsection{Polarized microscopy observations}

Xylitol crystals were observed in the air and in the molten supercooled xylitol by optical microscopy with cross polarisers (Leica DM 4500P). Supercooled xylitol was mounted on a glass slide and seeds were then added to the edge to trigger crystallization. This slide was placed on a Linkam LTS 350 heating stage to control the sample temperature. The temperature was checked with benzophenone and benzoic acid melted at a heating rate of $10 \mathrm{~K} / \mathrm{min}$. Deviations of 1.3 and $2.4^{\circ} \mathrm{C}$ were observed, respectively, between the melting temperature observed and the certified temperature. That makes possible to correct the real temperature with a linear regression. Micrographs were taken periodically to check the crystal growth with rates found in the literature. Additionally, crystallized samples after seeding and shearing were observed.

\subsubsection{DSC measurements}


DSC was employed to determine the melting enthalpy and melting temperature of the different sheared samples. A Netzsch 200 F3 Maia differential scanning calorimeter was employed. The calibration and experimental characteristics used were: 1) temperature calibration performed with $\mathrm{Hg}, \mathrm{H}_{2} \mathrm{O}$, Ga, In, Sn, Bi and $\mathrm{CsCl}$; 2) heat flow calibration using sapphire; 3) sample weight: $11-17 \mathrm{mg}$, weighed by a Mettler Toledo AB-135S balance (resolution: $0.01 \mathrm{mg}$, repeatability precision: $0.03 \mathrm{mg}$ ); 4) aluminium sealed crucibles; 5) heating rate: $0.5 \mathrm{~K} / \mathrm{min}$. According to the procedure proposed by Lazaro et al. [25], DSC measurements should be at a thermal equilibrium rate, previously obtained on the sample. Successive heating and cooling are performed on the sample, halving the heating and cooling rate in each cycle. The aim is to find the rate when the thermal equilibrium is reached. Nevertheless, in this case, as the sample polymorphism must be kept unchanged, thermal equilibrium tests could not be performed. Thus, a heating rate of $0.5 \mathrm{~K} / \mathrm{min}$ was selected in contrast to $1 \mathrm{~K} / \mathrm{min}$ selected by [26].

\subsubsection{X-Ray diffraction experiments}

$\mathrm{X}$-ray powder diffraction (XRPD) measurements were carried out to compare the diffraction patterns between the fresh xylitol and the sheared xylitol samples. Diffractograms were obtained with a D-Max Rigaku, Ru300 diffractometer, provided with a cupper rotating anode. The diffractometer works at $40 \mathrm{kV}$ and $80 \mathrm{~mA}$ and a graphite monochromator is used to select $\mathrm{Cu}$ $\mathrm{K} \alpha$ radiation. A step size of $2 \theta$ of 0.03 in the angular range from 5 to $40^{\circ}$ with $0.5 \mathrm{~s}$ per step was used.

\subsubsection{Thermogravimetric measurements}

Thermogravimetric analysis (TGA) was carried out using a TGA from TA Instruments, model SDT 2960. The weight changes in samples about $10 \mathrm{mg}$ were measured under a controlled air atmosphere at a heating rate of $10 \mathrm{~K} / \mathrm{min}$ from ambient up to $400^{\circ} \mathrm{C}$.

\section{Results and discussion}

\subsection{Description of pristine seeds}

Figure 2 shows a microscopic picture of the sieved xylitol crystals in air as supplied by the manufacturer to observe their shape and appearance. This picture shows chunks of crystalized xylitol after sieving. Apparently, these seeds are polycrystals formed by crystals oriented in different directions and aggregated in a single object. Fine xylitol crystalline dust (or crystal) is attached to the surface of the polycrystal, producing a rough texture. The micro-crystalline dust could be washed off the seed crystal surfaces and become viable growing crystals in the supercooled molten xylitol. The microcrystalline dust particles on the seed surfaces are commonly created when crystals are dried after their preparation or by microattrition, which occurs during the handling of the dried crystals. The forces holding the microcrystalline dust on the seed crystal surfaces are generally weak (van der Waals) attractive forces, thereby permitting their easy release when the crystals are placed into the crystallization medium [27]. These seeds to be added in the molten xylitol is going to be called throughout the article as "parent seed cluster". 


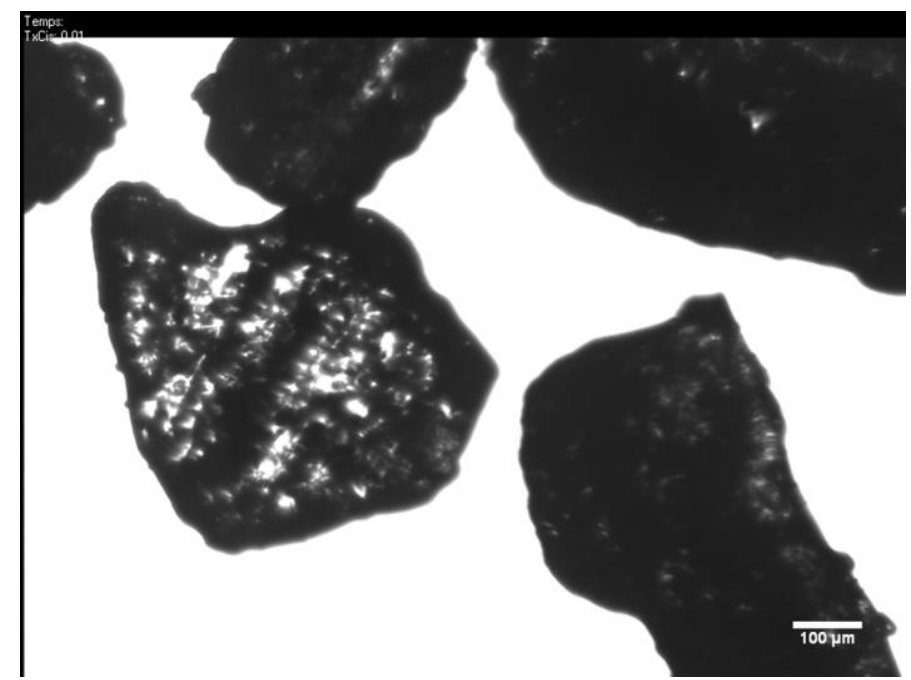

Figure 2. Sieved xylitol crystals in air at room temperature.

\subsection{Description of seeds in molten supercooled xylitol}

276 Sieved xylitol crystals were used as seeds and introduced in the supercooled xylitol sample at

$27790^{\circ} \mathrm{C}$ in the counter-rotating shear cell. This observation was made at a very low shear rate,

278 simply to observe how the parent seed cluster looked like.

279 Figure 3A shows one of the parent seed cluster observed while rotating at very low shear rate.

280 The parent seed cluster looks like an aggregate of crystals presenting flat faces at the outside

281 surface of the cluster. There are different reasons which could explain that the two seeds looked

282 different in figures 2 and 3 . The crystal in figure 3 is larger in one of its dimensions, maybe as a

283 consequence of the sieving process. While the counter-rotating shear cell is in operation, it takes

284 time to find one of the added parent seed cluster. Hypothetically, this could give time for the

285 outside crystals of the parent seed cluster to grow and then be fragmented into crystals by means

286 of shear and serve as secondary nucleation sites for crystallization. In addition, the low shear

287 induces the detachment of the fine crystalline dust (crystals with a much smaller size) from the

288 crystal surface when suspended in the molten xylitol. The fine crystalline dust could promote

289 crystallization by an initial breeding mechanism, as explained in the introduction section [13-

290 16]. It should be pointed out that the faceted crystal depicts some cracks, indicated with an 291 arrow in figure 3A.
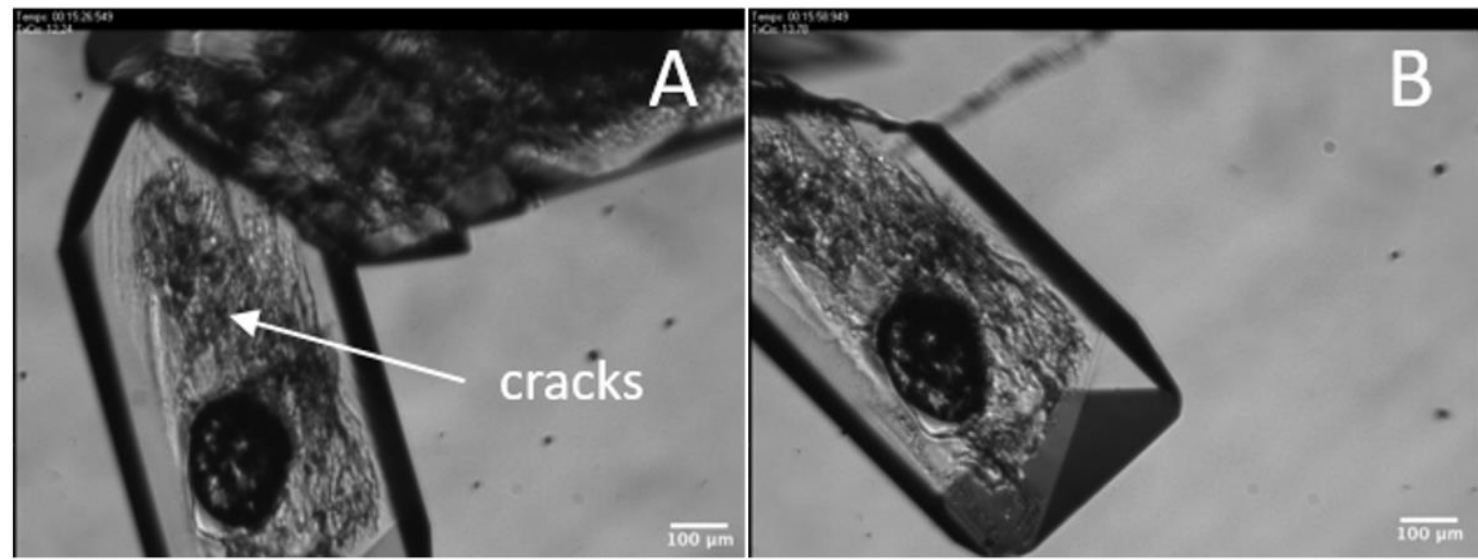
Figure 3. A: Sieved xylitol crystals used as seed suspended in the supercooled xylitol at $90^{\circ} \mathrm{C}$, no shear is applied. B: Crystal agglomeration and mechanical separation when sheared.

Figure 4 illustrates the growth of the outside layer of monocrystals of a sieved seed suspended in supercooled xylitol at $70^{\circ} \mathrm{C}$ with no shear. Given that the observations made by polarized microscopy were carried out close to the temperature limit between two distinct growth patterns, it is not possible at this scale to distinguish whether either layer-by-layer growth without nucleation on the surface or a branched growth has taken place. These crystals are quite similar to those shown in Zhang et al. [28]. The crystallization rate at $70^{\circ} \mathrm{C}$ is consistent with the published data found in the literature [3].
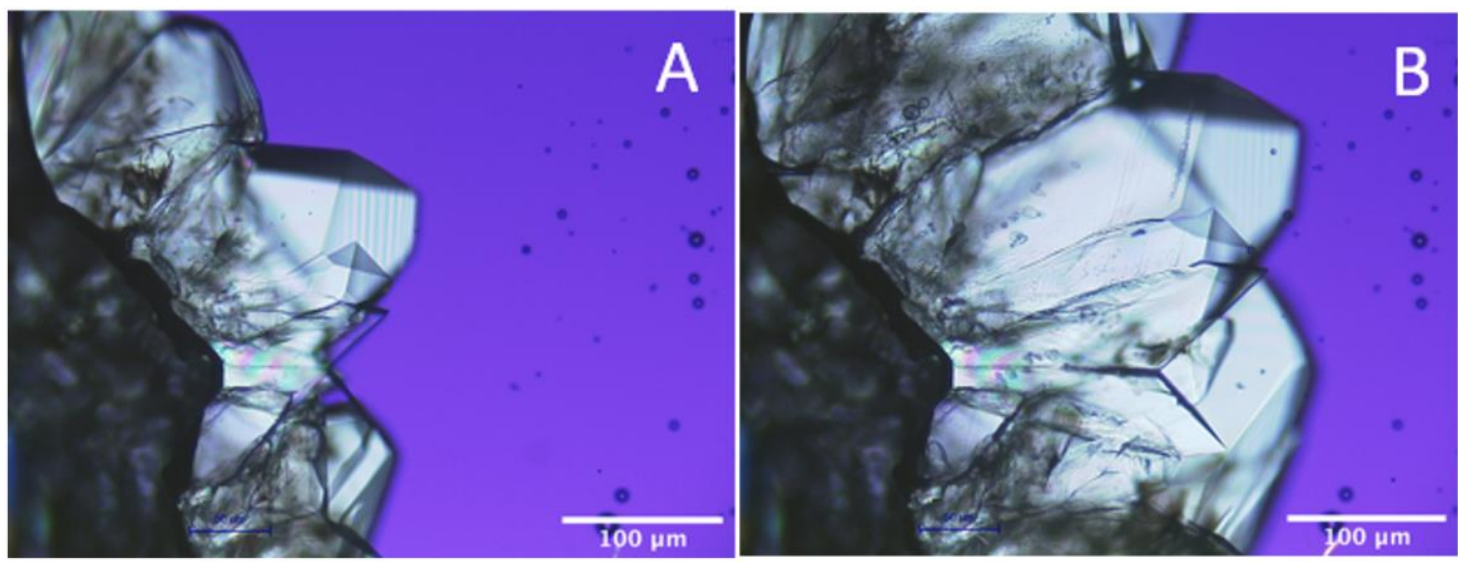

Figure 4. Xylitol growth at $70^{\circ} \mathrm{C}$ with a time difference of $40 \mathrm{~s}$ between $\mathrm{A}$ and $\mathrm{B}$. No shear is applied.

\subsection{Effect of shear on seeds}

It was checked that crystallization did not occur in a reasonable time from the point of view of a short-term TES application, if no seed was added even with shear (crystallization was not observed during an experiment of 66 hours), nor if seeds were added but no shear was applied. For that reason, the combined techniques of seeding and shearing was adopted.

Observations of the shear process on the seeded xylitol sample suggest that:

1) In the case of dispersion mechanisms:

-very low shear rate removes the crystalline dust observed on the initial seed,

-while increasing the shear rate, erosion can take place, resulting in the continuous detachment of crystals from the outer surface of the parent seed cluster as a result of fluid-induced stresses acting upon them,

-increasing the shear rate further should lead to the rupture of the parent seed cluster resulting in its deagglomeration into smaller aggregates or individual crystals. The breakage of agglomerates is critically influenced by two opposing factors, namely the mechanical strength of the agglomerate and the breaking forces [10]. Results obtained by one of the authors of this article showed that the critical shear stress (and thus critical shear rate) for shear-induce rupture 
of carbon black aggregates in a molten polymer matrix depends on the size of the agglomerates [29].

323 2) In the case of shear-induced aggregation:

324 -very low shear rates may boost aggregation since, when collision takes place, there is a longer contact time between the crystals,

-when increasing the shear rate, the frequency of collision is greater but less efficient from the point of view of contact time for subsequent aggregation. The effect of shear on the probability of collision and time of contact was discussed by on the authors of the article in the case of shear-induced coalescence in immiscible polymer blends [30]. Large shear rates also induce the crystal alignment along the shear direction. This was also observed in crystallization of "models system" [31].

The effect of shear rates of different magnitudes is illustrated in figures 3 to 6 .

Figure 3 shows the detachment of a monocrystal from the parent seed cluster when the shear rate was increased (figure 3B). These aggregates can be slightly porous, allowing penetration of the melted xylitol and promoting this detachment under shear.

Figure 5 shows the result of the application of quite low shear rate, where faceted crystals can be seen while the sample was sheared at a higher shear rate than the one initially applied to find the parent seed cluster. These crystals could be either eroded fragments (crystals detached from the outer surface of the parent seed cluster), disaggregated fragments (resulting from the rupture of the parent seed, an unlikely phenomenon since it was never observed at this shear rate), or crystal nuclei which have grown from the detached crystalline dust at lower shear rate. It is difficult to opt for one of these suppositions, as the crystalline dust attached to the seed crystal prevents a clear observation of how the parent seed cluster is constituted.

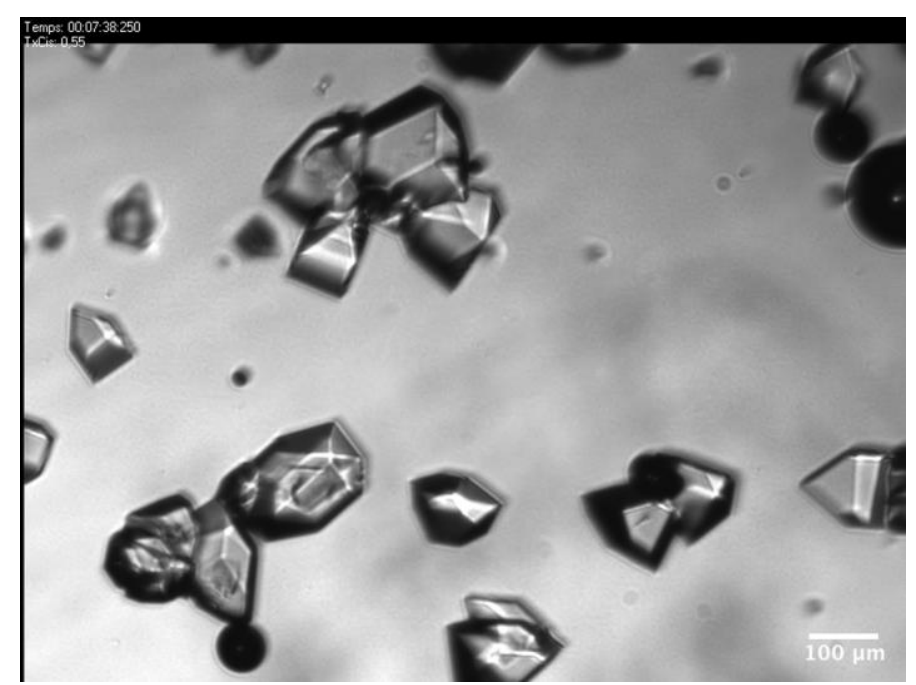

Figure 5. Microscope observations at $90^{\circ} \mathrm{C}$ after the sample has been sheared at $3 \mathrm{~s}^{-1}$.

346 It was also observed at $90^{\circ} \mathrm{C}$, that at shear rates above $20 \mathrm{~s}^{-1}$, very small crystals immediately 347 started to flow, as shown in figure 6 (see the background of figure 6A). These small crystals 
could result from the rupture or deaggregation of the parent seed cluster above a critical shear stress.

350 If it is assumed that the sieved crystals serve as nucleation sites for secondary nucleation and 351 further crystallization, it is thus important to control the total crystalline surface available. One 352 strategy could be to shear at high shear rates (higher than the critical shear rate for rupture of the 353 initial seeds to have a large number of separated "small crystals") and then to diminish the shear 354 rate to induce the xylitol crystallization. In light of these results, and bearing in mind the use of 355 a stirred tank as the TES system, the key parameters from the point of view of the xylitol seeds 356 would be the crystal volume fraction, the cohesion strength of the seed cluster and the size and 357 shape of the monocrystals after deaggregation. The critical shear stress to be applied to 358 deaggregate the parent seed cluster should depend on its initial size [29]. Zhang observed that 359 xylitol crystalline grains were usually damaged, giving rise to many surface nucleation sites. He 360 measured the xylitol growth speed when crystalline grains were dropped into supercooled 361 xylitol. To smooth the granular surfaces, the temperature was tuned up and down iteratively 362 with the microscope hot stage being around the melting temperature of xylitol in order to 363 prepare seeds with a simpler geometry [28]. From the point of view of the application, the 364 seeding technique coupled to shear should ensure reproducibility in crystallization and among 365 the stored energy discharge tests.

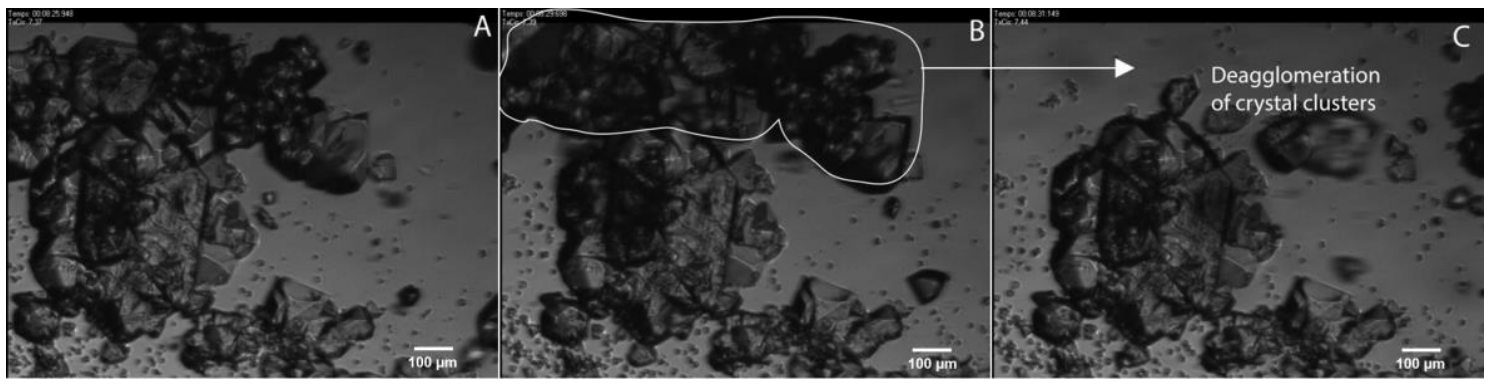

367gure 6. Microscope observations at $90^{\circ} \mathrm{C}$ when shearing at $20 \mathrm{~s}^{-1}$. Time difference from A: 4 seconds for B and 6 seconds for $\mathrm{C}$.

\subsection{Effect of shear on xylitol crystallization}

370 In parallel to local rheo-optical observations, which allowed to understand the effect of shear on

371 xylitol seeds, rheological measurements were performed as another way to probe xylitol 372 crystallization.

373 As already explained, the sample was loaded in three steps. First, half of the sample was loaded on the Peltier plate and thermostabilization at $90^{\circ} \mathrm{C}$ for 20 minutes without shear was executed;

375 next, a parent seed cluster was loaded on the supercooled xylitol representing an average mass 376 fraction at a percentage of $5 \cdot 10^{-3} \%$ (standard deviation of $57 \%$ ); and finally, the other half of the 377 xylitol preheated at $90^{\circ} \mathrm{C}$ in an oven was loaded. It must be highlighted that in addition to the 378 high deviation in mass or mass fraction among different seeds, the mass of one seed is in the 379 limit of the precision balance $(0.03 \mathrm{mg})$. Once the sample was loaded, the viscosity at different 380 shear rates was measured over time. Figures 7a, 8a and 9a shows a succession of viscosity 381 changes when different xylitol samples were sheared at 5,10 and $15 \mathrm{~s}^{-1}$. Each shear rate was 
tested on three different fresh samples. The results were not repeatable. This was expected, as neither was their radial position in the parallel plate geometry within the supercooled xylitol well controlled, nor were identical the added parent seed cluster (in terms of mass). The position (along the radius) in the shear cell determines the applied local shear rate and thus the final crystal size distribution. The seed mass and the applied local shear rate should determine the seed surface distribution, which is directly related to the amount of nucleation sites.

The reproducibility of the measurements was tried to be improved by imposing a pre-shear (shear rate larger than the critical condition for rupture of the parent seed cluster). But this did not help, since the critical point is the uncertainty and high deviation on the mass among the initial parent seed clusters. This operational strategy should be researched in future work. Only direct measurements (without that pre-shear) are commented below. Tests were also performed at lower temperatures $\left(70\right.$ and $\left.80^{\circ} \mathrm{C}\right)$. They are not reported, since in those cases crystallization was almost spontaneous.

What is common to all the tests is a first zone with a constant viscosity, which fits with the previous measured viscosity of the supercooled xylitol reported by Palomo del Barrio et al. [32]. The low quantity of the parent seed cluster does not affect the viscosity of the molten xylitol. Then there is a second zone with a viscosity jump, due to the increase of the solid volume fraction as result of the crystallization process. The viscosity jump is sudden and the viscosity quickly increases from 0.5 to around $10 \mathrm{~Pa} \cdot \mathrm{s}$. However, it could not be evidenced any relationship between this "induction time" and the shear rate, due to the explained non-identical seeding conditions. In most samples, a third zone with a change to a lower slope of the viscosity-time curve is observed. The viscosity curve presents some oscillations at the slope modification, which seems to always happen around $10 \mathrm{~Pa} \cdot \mathrm{s}$ whatever the shear rate (for the rate of investigation). This is especially noticeable at 5 and $10 \mathrm{~s}^{-1}$. The solid volume fraction (linked to the crystallization process) corresponding to this level of viscosity can be estimated using the Krieger-Dougherty model for suspension [33] (equation 1) and considering spherical entities:

$$
\eta_{\text {susp }}=\eta_{m} \cdot\left(1-\frac{\phi}{\phi_{\max }}\right)^{-[\eta] \cdot \phi_{\max }} \text { (eq. 1) }
$$

where $\eta_{\text {susp }}$ is the viscosity of the suspension, $\eta_{m}$ is the viscosity of the suspending medium, i.e. the viscosity of the molten xylitol at $90^{\circ} \mathrm{C}(0.5 \mathrm{~Pa} \cdot \mathrm{s}), \phi$ is the solid volume fraction in the suspension, $\phi_{\max }$ is the maximum packing fraction and $[\eta]$ is the intrinsic viscosity of the system.

The values of $\phi_{\max }$ and $[\eta]$ depend on the type, shape and size polydispersity of the suspended particles. The maximum packing fraction also varies with shear [34]. Due to the absence of reproducibility and quite narrow range of investigated shear rates, it was not tried to be detected any effect of the shear rate on the solid fraction. Similar parameters as the ones used on crystallization of a different system in a previous work of some of the authors were used [18]. Considering monodisperse spheres $\left(\phi_{\max }=57 \mathrm{vol} \%,[\eta]=2.67\right.$ and a low shear rate [35]), an apparent volume fraction around $50 \mathrm{vol} \%$ of solid phase was estimated for this level of viscosity 
$420(10 \mathrm{~Pa} \cdot \mathrm{s})$. In comparison, using parameters for random suspension of aggregates $\left(\phi_{\max }=38\right.$

$421 \mathrm{vol} \%,[\eta]=2.4$ and a low shear rate [36]), an apparent volume fraction around $35 \mathrm{vol} \%$ of solid 422 phase was obtained. Whatever the model used, the estimated apparent solid volume fraction

423 appears to be close to the maximum packing fraction. It means that aggregated crystals already

424 form a quite packed crystalline network with a lot of interactions between aggregates for this

425 range of viscosity.

426 The normal force evolution was also recorded in parallel to the viscosity evolution and shown in 427 figures $7 \mathrm{~b}, 8 \mathrm{~b}$ and $9 \mathrm{~b}$. A normal force jump is also recorded. The normal force jump is always 428 recorded with some delay compared to the viscosity one. It seems to be concomitant with the 429 change of slope and the presence of oscillations in the viscosity curve around $10 \mathrm{~Pa} \cdot \mathrm{s}$. It is noted 430 that positive normal force values were recorded in contrast to assumed negative values as a 431 consequence of the volume shrinkage during crystallization. Due to the large volume fraction 432 present at that moment, it is assumed that positive normal forces arise from the rearrangement of 433 the crystalline aggregates inside the crystalline network which tends to develop under shear, 434 [Personal communication with Carlos Gracia, from TA Instruments]. As the crystalline fraction 435 increases, the crystalline network becomes more and more packed and breakage and 436 rearrangement of the aggregated structures inside the network may happen under shear in order 437 to reach a more packed structure [18].

438 The normal force increases and levels off around 60N (limit of the normal force transducer), 439 where oscillations are recorded. All these rheological measurements should be taken with 440 caution because slippage can happen in addition to breakage and rearrangement of the 441 crystalline network at such high levels of solid volume fraction under shear.
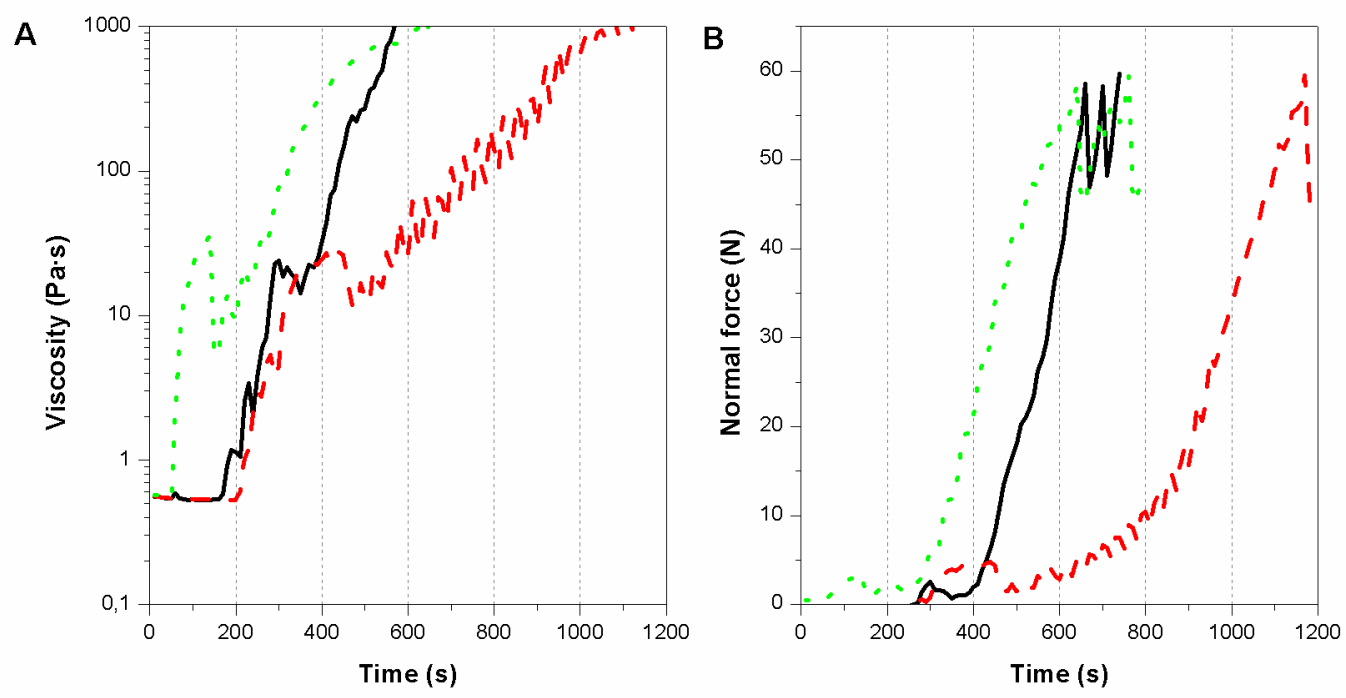

-Measurement 1 - Measurement $2 \ldots$ Measurement 3

Figure 7. Viscosity and normal force evolution of supercooled xylitol at $90^{\circ} \mathrm{C}$ under the application of a shear rate of $5 \mathrm{~s}^{-1}$. 

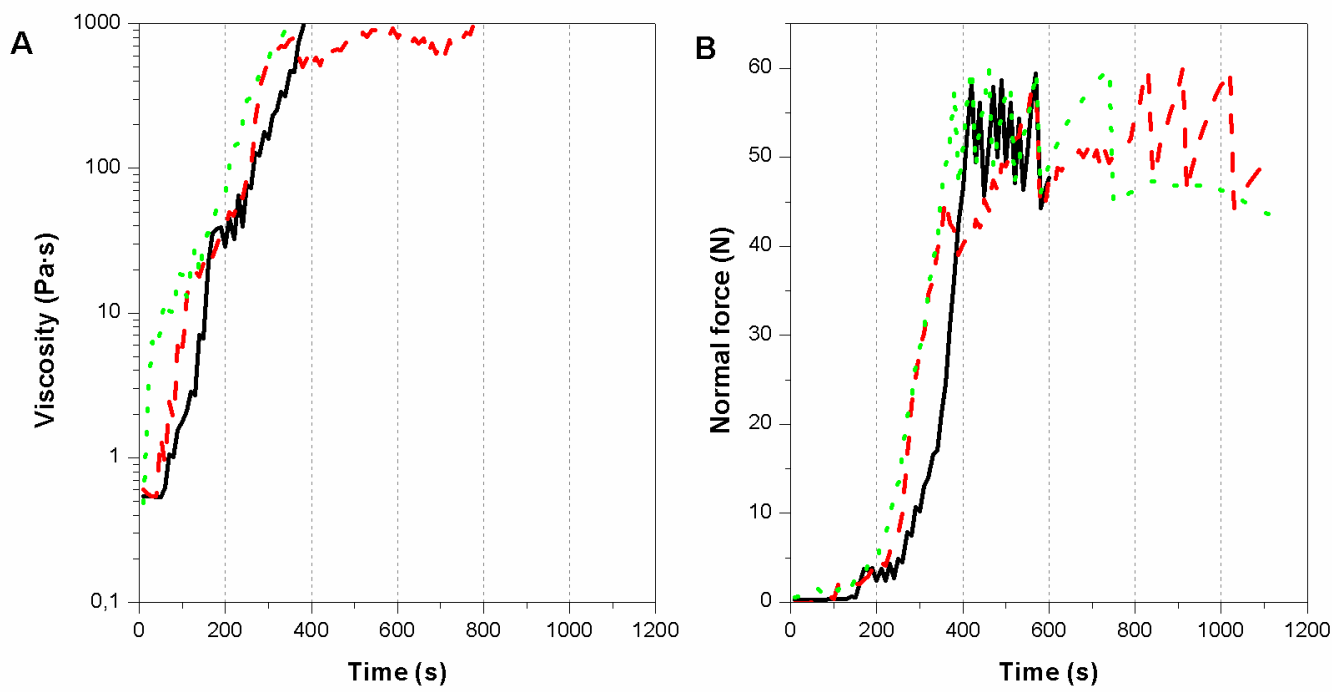

—Measurement $1--$ Measurement $2 \cdots$ Measurement 3

Figure 8. Viscosity and normal force evolution of supercooled xylitol at $90^{\circ} \mathrm{C}$ under the application of a shear rate of $10 \mathrm{~s}^{-1}$.
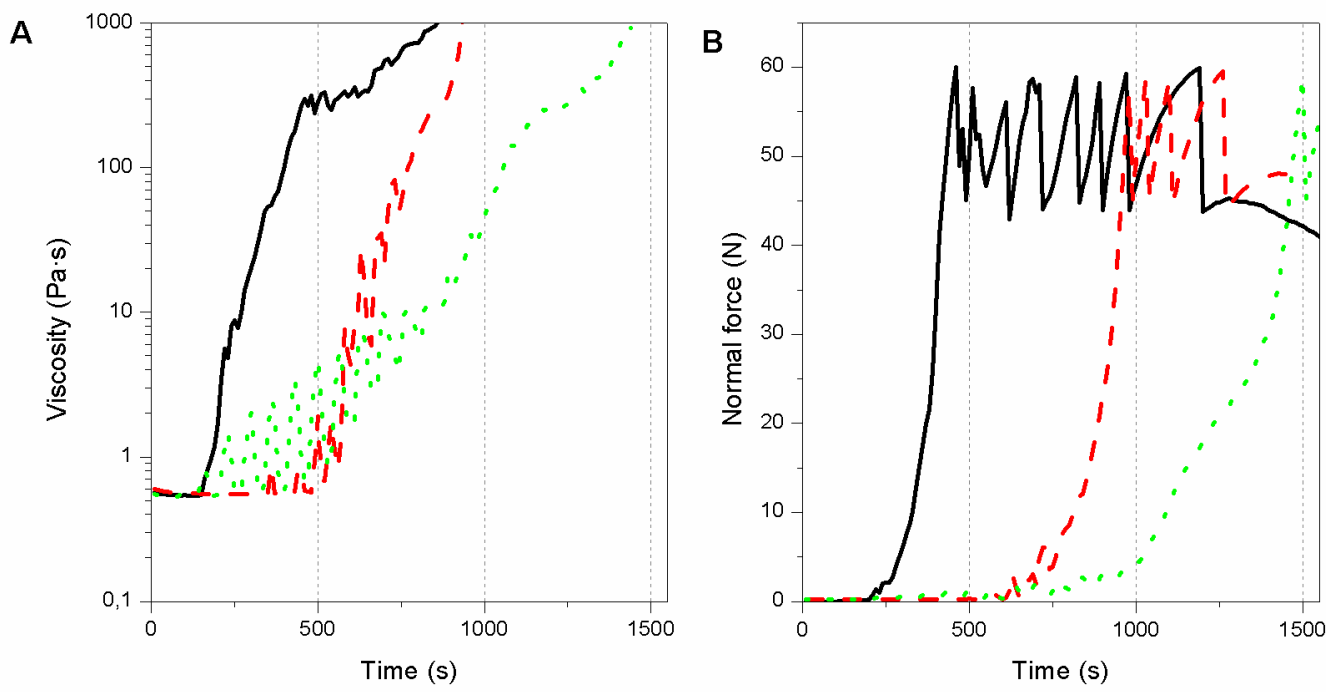

Figure 9. Viscosity and normal force evolution of supercooled xylitol at $90^{\circ} \mathrm{C}$ under the application of a shear rate of $15 \mathrm{~s}^{-1}$.

The viscosity and normal force evolutions are indirect signatures of the complex crystallization process under shear with characteristics features, such as the viscosity jump, the normal force jump and the level-off of the normal force. It was considered that the times related to these different features are indicative of the crystallization process. The viscosity jump gives a first idea of the onset of crystallization (overestimated, since crystallization happens earlier and is not detected by the viscosity measurement). The normal force jump should correspond to some first reorganization of the crystalline aggregates. The level-off of the first normal force can be considered as an indication of an almost complete crystallization, although the viscosity curve is still evolving. 

to better characterize the crystallization under shear. Due to the high level of noise from the viscosity curve, an onset time for crystallization as indicated by the viscosity signature was estimated. The mathematical criterion adopted in this case is when the first derivative reaches a value higher than 0.005 . From the normal force measurements, an onset time was also estimated. This onset time was calculated as the intersection point between the baseline (normal force equal to 0 ) and the tangent at the inflection point. To find those inflection points, the curves were slightly smoothed to remove the noise and make possible its estimation. Next, the normal force curve, which shows initially an exponential growth, was fitted to a Gaussian function (equation 2). According to the fitting, the time when the Gauss bell reaches its maximum was considered as an estimate of a "complete" (almost complete) crystallization time. This peak value for the normal force ranges between 50 and $60 \mathrm{~N}$ ( $60 \mathrm{~N}$ is the maximum normal force allowed by the transducer). Rheological measurements after that moment should be considered as invalid, as the limit of the normal force is attained and the gap increases to avoid the damage of the device. The fitting parameters and the coefficient of determination are compiled in table A.1 from Appendix A.

$$
F_{n}=F_{n 0}+\frac{A}{w \cdot \sqrt{\frac{\pi}{2}}} \cdot e^{\frac{-2 \cdot\left(t-t_{c}\right)^{2}}{w^{2}}}(\text { eq. 2) }
$$

Figure A.1 in Appendix A shows these mathematical fittings on one of the curves per shear rate as an example. And table 1 displays the two onset times and the complete crystallization time obtained according to the procedure just explained, as well as the average time of the three measurements per shear rate and its standard deviation. In light of the results, there is a high deviation among the measurements, although they were executed under the same conditions of shear. This is another way to see the non-reproducibility. This leads us to reaffirm the assumption that a good control over the seeding process is crucial. Because of the non-reproducibility, it is impossible within the range of investigated shear rates to demonstrate any evidence of the effect of the magnitude of the shear rate on the characteristic crystallization times. But the interesting point is to compare the order of magnitude of these crystallization times estimated with combined seeding and shearing relatively to the crystallization after seeding without shear. This will be discussed in the following.

488 These crystallization times are of the same order of magnitude as crystallization times in the rheooptical shear experiments. The exact comparison cannot be done because in the counter-rotating shear cell, the objective is to follow the behaviour of one parent seed cluster, for which the shear rate must sometimes be slightly modified in order to keep it in the window of observation.

492

\begin{tabular}{|c|c|c|c|c|c|c|}
\hline & \\
\hline & Shear rate & M1 & M2 & M3 & Average & SD \\
\hline Onset time (s) (viscosity curve) & & 170 & 210 & 50 & 143 & 83 \\
\hline Onset time ( $s)\left(F_{n}\right.$ curve $)$ & $5 \mathrm{~s}^{-1}$ & 462 & 774 & 293 & 510 & 244 \\
\hline Complete crystallization time $(s)\left(F_{n}\right.$ curve) & & 789 & 1206 & 617 & 871 & 303 \\
\hline
\end{tabular}




\begin{tabular}{|c|c|c|c|c|c|c|}
\hline Onset time (s) (viscosity curve) & \multirow{3}{*}{$10 \mathrm{~s}^{-1}$} & 60 & 40 & 10 & 37 & 25 \\
\hline Onset time (s) ( $F_{n}$ curve $)$ & & 287 & 223 & 173 & 228 & 57 \\
\hline Complete crystallization time (s) $\left(F_{n}\right.$ curve) & & 503 & 376 & 475 & 451 & 67 \\
\hline Onset time (s) (viscosity curve) & \multirow{3}{*}{$15 \mathrm{~s}^{-1}$} & 150 & 310 & 150 & 203 & 92 \\
\hline Onset time ( $(s)\left(F_{n}\right.$ curve $)$ & & 331 & 770 & 954 & 685 & 320 \\
\hline Complete crystallization time (s) ( $F_{n}$ curve) & & 479 & 1097 & 1553 & 1043 & 539 \\
\hline
\end{tabular}

$493 \quad$ Table 1. Onset and complete crystallization times according to the shear conditions.

494 A similar experiment was performed but applying a very low shear rate defined as the minimum

495 allowed one by the rheometer to record an acceptable angular velocity in order to simulate

496 crystallization after seeding but at rest conditions. The aim of this experiment was to quantify the

497 improvement of the combined technique of seeding and shearing in comparison with only

498 seeding. The shear rate to emulate this non-shear or rest condition was $0.01 \mathrm{~s}^{-1}$. Based on the

499 results shown in figure 10, the crystallization as seen via the normal force response follows a

500 linear growth according to the fitting function defined by equation 3.

501

$$
F_{n}=-0.22402+0.00331 \cdot t(\text { eq. 3) }
$$

502 The complete experiment is not shown, since as the crystallization progresses, the control of the

503 low shear rate by the control stress rheometer was more difficult. The rheometer was not able to

504 maintain the constant shear rate conditions. If the linear fit is extrapolated to a normal force up to

$50560 \mathrm{~N}$, which was visually checked as the end of xylitol crystallization, the complete crystallization

506 time can be estimated, being 303 minutes. The onset time for this experiment was not estimated

507 since there would be large errors on this value due to the noisy signal. This estimated time of 302

508 minutes to crystallize a seeded sample at rest is much larger than any of the complete

509 crystallization times determined from experiments under shear $\left(14.5 \pm 5 \mathrm{~min}\right.$ at $5 \mathrm{~s}^{-1}, 7 \pm 1 \mathrm{~min}$ at

$51010 \mathrm{~s}^{-1}, 17 \pm 9 \mathrm{~min}$ at $15 \mathrm{~s}^{-1}$, see Table 1 ). Based on the present data, the crystallization rate appears

511 to be increased by a factor of around 23 by means of the shearing technique and adding a mass

512 fraction of seeds of only $5 \cdot 10^{-3} \%$.

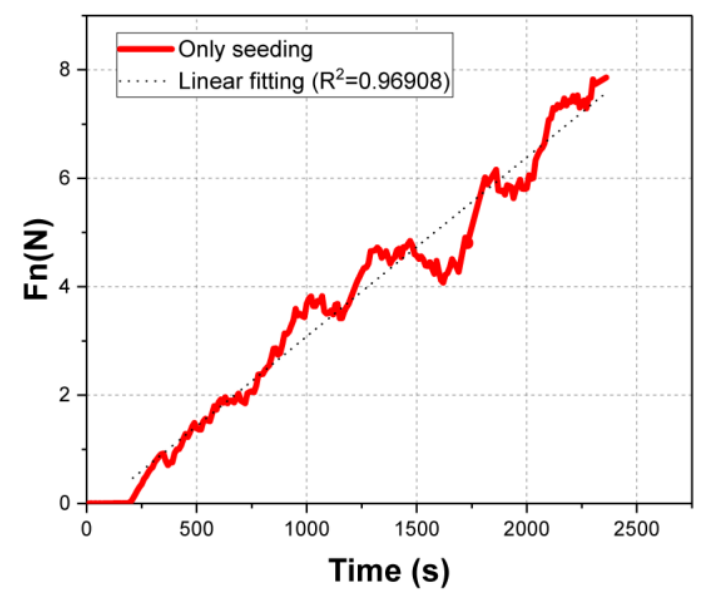


Figure 10. Procedure to estimate the crystallization time from the normal force evolution at rest conditions (shear rate $=0.01 \mathrm{~s}^{-1}$ )

516 Figures 11a and 11c show samples sheared at low shear rates with the counter-rotating shear 517 cell at $90^{\circ} \mathrm{C}\left(10 \mathrm{~s}^{-1}\right.$ at the edge of the shear cell and $100 \mathrm{~s}^{-1}$ at the central position of the shear cell, 518 which means a low shear rate zone due to the plate-plate geometry). These micrographs show 519 the aggregation of large crystals. Figure $11 \mathrm{~b}$ shows a sample sheared at $100 \mathrm{~s}^{-1}$ and taken from 520 the edge position of the plate-plate geometry, having experienced a higher shear rate than the 521 previous samples. In this case, an aggregation of smaller crystals is observed.
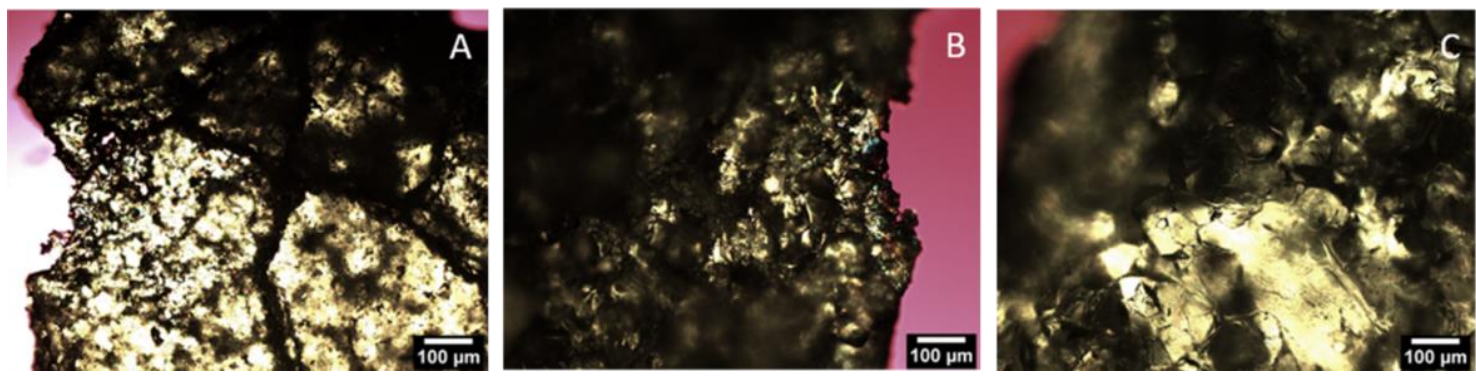

523Figure 11. Micrographs of different xylitol samples sheared at $90^{\circ} \mathrm{C}$. A: $100 \mathrm{~s}^{-1}$ center position; B: $100 \mathrm{~s}^{-1}$ 524 edge position; $\mathrm{C}: 10 \mathrm{~s}^{-1}$ edge position.

\section{$525 \quad 3.5$ Crystalline structure}

526 The melting temperature and latent heat for the fresh xylitol and xylitol sheared at different 527 velocities with the rheometer were taken when the samples were fully crystallized. These are 528 shown in table 2. There are no significant differences between the fresh xylitol and the sheared 529 xylitol, as can be observed in figure 12. This is consistent with other results reported in the 530 literature [2]. It can be assumed that at these shear rates sample crystallinity is preserved.

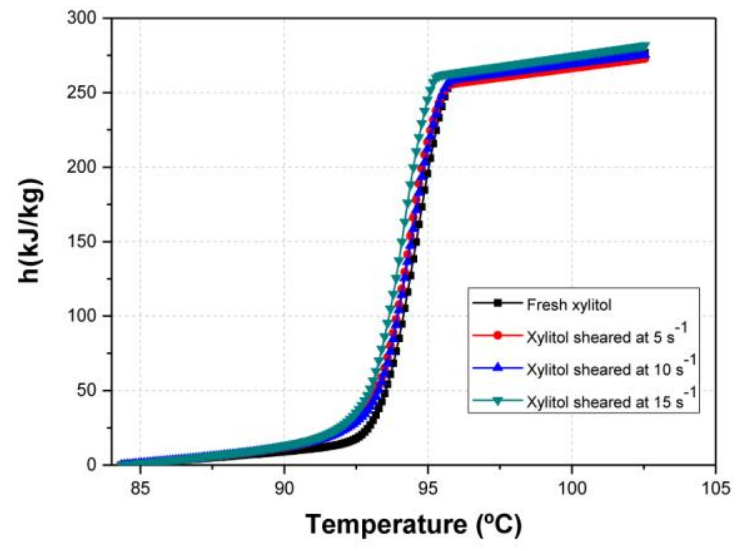

Figure 12. Enthalpy-temperature curve for fresh and sheared xylitol obtained with the DSC procedure proposed by Lazaro et al. [25] 


\begin{tabular}{lllll} 
Non-sheared xylitol & 16.78 & 241.2 & 92.8 & 94.7 \\
\hline Sheared xylitol at $5 \mathrm{~s}^{-1}$ & 14.48 & 233.6 & 92.7 & 94.4 \\
\hline Sheared xylitol at $10 \mathrm{~s}^{-1}$ & 14.27 & 236.3 & 92.8 & 94.5 \\
\hline Sheared xylitol at $15 \mathrm{~s}^{-1}$ & 11.27 & 238.2 & 92.5 & 94.1
\end{tabular}

$534 \quad$ Table 2. Latent heat and melting temperature for fresh and sheared xylitol

535 Diffractograms of pure xylitol supplied by Sigma Aldrich and of sheared samples were

536 compared to each other as well as with available data in the literature [37]. Figure 13 shows the

537 diffraction patterns of the bulk xylitol and the sheared xylitol. They are identical and consistent

538 with the data in the literature. Therefore, it can be concluded that no monoclinic phase (or

539 disappearing polymorph, as named by Dunitz and Bernstien [38]) was formed on the solid

540 phase. When polymorphic forms of a substance occur, intentional seeding with one of the

541 polymorphs is a useful and often the most successful way of preferentially producing it rather

542 than the other.

543

544

545

546

547

548

549

550

551

552

553

554

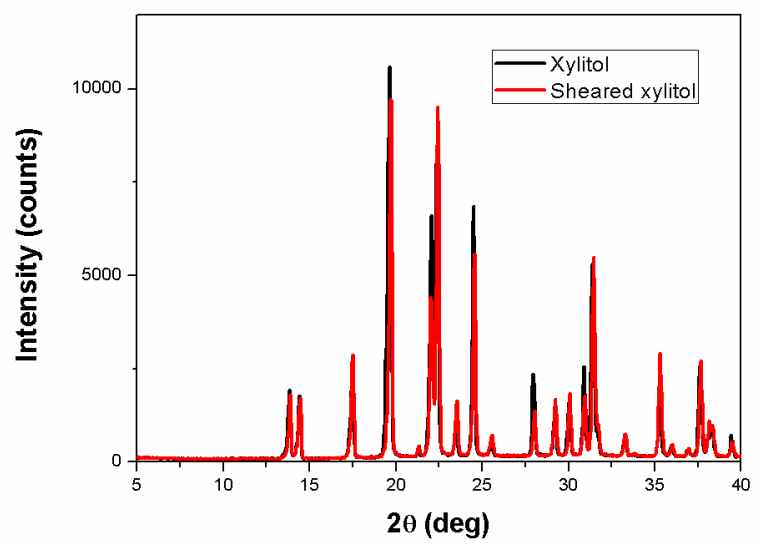

Figure 13. XRPD diffractograms for xylitol supplied by Sigma Aldrich and after shearing at 100

$$
\mathrm{s}^{-1} \text { at } 90^{\circ} \mathrm{C} \text {. }
$$

\subsection{Thermal stability}

The thermal stability of the xylitol (both sheared and non-sheared) is a significant factor which would determine the performance of the TES system, where xylitol would be incorporated. The thermogravimetric analysis shows the mass loss of the sample as a function of temperature. As seen from TGA thermograms shown in figure 14, there are not changes in the degradation process between the sheared and non-sheared samples, where xylitol loses a large fraction of its mass at one step in about 200 and $330^{\circ} \mathrm{C}$. It can be stated that the initial temperature of the thermal degradation is much higher than the working temperature range of xylitol in TES applications. 


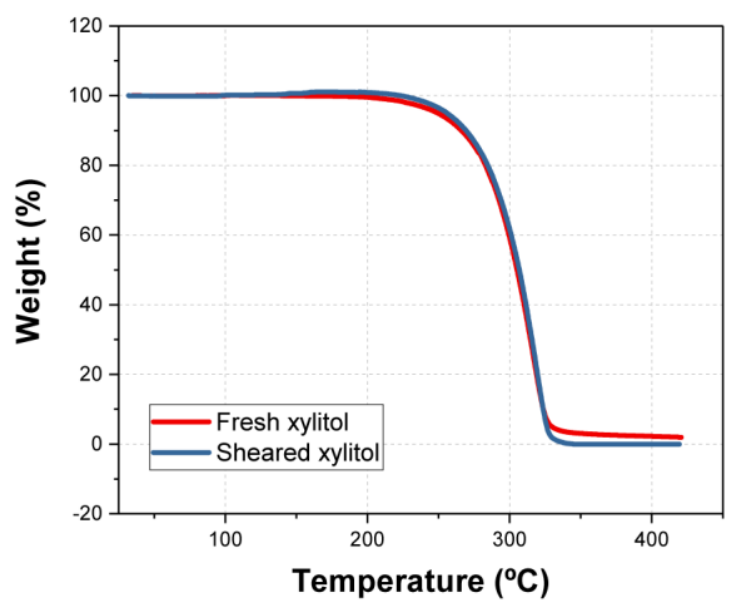

Figure 14. TGA thermograms for sheared and non-sheared xylitol.

\section{Conclusions}

Seeding in combination with shearing has been proposed as a promising technique to overcome the nucleation triggering and low crystallization rates of xylitol to be used as a PCM in shortterm TES applications. In particular, this article aims at providing a better understanding of these processes by means of rheo-optical and rheometry techniques in isothermal supercooling conditions.

The rheo-optical approach allows to observe the mechanisms happening to a single parent seed cluster suspended in the molten xylitol submitted to shear. Different mechanisms leading to the presence of many tiny crystals in the molten supercooled xylitol were identified under the action of shear:

- Fine crystalline dust attached to the surface of the crystal is first released.

- Next, the parent cluster is dispersed by rupture and erosion mechanisms depending on the applied shear rate.

The crystal fragments become potential secondary nucleation sites favouring further crystallization of xylitol. The surface developed by the crystal fragments (resulting from the parent seed cluster dispersion) should be one of the key parameters to control the kinetics of crystallization under shear.

Crystallization induced by a combined action of seeding and shearing was also investigated by rheometry. Characteristic crystallization times, "onset" and "almost complete" crystallization times, could be estimated from the evolution of the viscosity and normal force respectively.

- The combined technique of seeding and shearing appears to be very efficient to trigger and accelerate the crystallization of xylitol with an average improvement of the crystallization time by a factor of around 20 compared to the one obtained in conditions of no shear (in presence of seeding).

- It should be noticed that this result is observed even if rheological measurements were not reproducible. This is due to the fact that the present seeding and shearing protocols 

fragments.

Post-mortem DSC and XPRD analyses of the fully crystallized seeded and sheared samples did not show any effect on the xylitol crystallinity degree.

In light of these results, the design of a stirred-tank prototype with xylitol will be a logical continuation of the present work. This research suggests that the control of the seeding under shear (controlled introduced seed mass and defined pre-shear protocol) could be a key point to control the crystallization kinetics. The bigger size of the prototype should overcome limits associated with the small volume in the rheometer and allow the validation or invalidation of the hypothesis. Larger scale experiments will question the sustainability of the present solution in the next cycles of melting and crystallization 1) by ensuring the presence of remaining crystals after the melting step, with the subsequent decrease of the energy storage efficiency and the possible difficulties of temperature control, or 2) via the development of a seeds reservoir and injection system, which entails a more complex system.

\section{CRediT authorship contribution statement}

599 Mónica Delgado: Conceptualization, Data curation, Formal analysis, Funding acquisition,

600 Investigation, Methodology, Resources, Roles/Writing-original draft

601 Miguel Navarro: Conceptualization, Data curation, Formal analysis, Investigation,

602 Methodology, Resources, Writing-review \& editing

603 Ana Lázaro: Conceptualization, Formal analysis, Funding acquisition, Investigation,

604 Methodology, Project administration, Resources

605 Sévérine A.E. Boyer: Conceptualization, Formal analysis, Investigation, Methodology,

606 Resources, Supervision

607 Edith Peuvrel-Disdier: Conceptualization, Formal analysis, Investigation, Methodology,

608 Resources, Supervision

\section{Acknowledgments}

610 This work was developed in the frame of the research project ENE2017-87711-R, partially

611 funded by the Spanish Government (Energy Program), the Government of Aragon (Ref: T55-

612 17R), Spain, and the EU Social Fund (FEDER Program 2014-2020 "Building Europe from

613 Aragon"). This research was partially carried out by Mónica Delgado during a research stay at

614 the Centre de Mise en Forme des Matériaux (CEMEF)-CNRS-Mines Paris, funded by a grant

615 from Programa CAI-Ibercaja-Estancias de Investigación. The authors would like to

616 acknowledge the use of the Servicio General de Apoyo a la Investigación-SAI, Universidad de

617 Zaragoza. Special thanks to Jean-Marc Haudin and Romain Castellani for fruitful discussions.

\section{References}


619 [1] M. Grembecka, Sugar alcohols-their role in the modern world of sweeteners: a review, Eur. 620 Food Res. Technol. 241(1) (2015) 1-14.

621 [2] E. Palomo del Barrio, R. Cadoret, J. Daranlot, F. Achchaq, New sugar alcohols mixtures for 622 long-term thermal energy storage applications at temperatures between 70 and $100^{\circ} \mathrm{C}$, Sol.

623 Energy Mater. Sol. Cells 155 (2016) 454-468.

624 [3] G. Diarce, I. Gandarias, A. Campos-Celador, A. García-Romero, U.J. Griesser, Eutectic 625 mixtures of sugar alcohols for thermal energy storage in the $50-90^{\circ} \mathrm{C}$ temperature range, Sol.

626 Energy Mater. Sol. Cells 134 (2015) 215-226.

627 [4] S.N. Gunasekara, J. Chiu, V. Martin, P. Hedströmb, The experimental phase diagram study 628 of the binary polyols system erythritol-xylitol, Sol. Energy Mater. Sol. Cells 174 (2018) 248-

629262.

630 [5] A. Seppälä, A. Meriläinen, L. Wikström, P. Kauranen, The effect of additives on the speed 631 of the crystallization front of xylitol with various degrees of supercooling, Exp. Therm. Fluid 632 Sci. $34(5)$ (2010) 523-527.

633 [6] M. Duquesne, A. Godin, E. Palomo del Barrio, F. Achchaq, Crystal growth kinetics of sugar 634 alcohols as phase change materials for thermal energy storage, Energy Procedia 139 (2017) 315635321.

636 [7] S. Ur-Rehman, Z. Mushtaq, T. Zahoor, A. Jamil, M.A. Murtaza, Xylitol: a review on 637 bioproduction, application, health benefits, and related safety issues, Crit. Rev. Food Sci. Nutr. $638 \quad 55(11)(2015) 1514-1528$.

639 [8] A. Godin, M. Duquesne, E. Palomo del Barrio, F. Achchaq, P. Monneyron, Bubble agitation 640 as a new low-intrusive method to crystallize glass-forming materials, Energy Procedia 139 641 (2017) 352-357.

642 [9] M. Duquesne, E. Palomo del Barrio, A. Godin, Nucleation triggering of highly undercooled 643 xylitol using an air lift reactor for seasonal thermal energy storage, Appl. Sci. 9 (2019) 267-276.

644 [10] S. Salyan, S. Suresh, Multi-walled carbon nanotube laden with D-mannitol as phase change 645 material: Characterization and experimental investigation, Adv. Powder Technol. 29 (12) (2018) $646 \quad 3183-3191$.

647 [11] S. Salyan, S. Suresh, Study of thermo-physical properties and cycling stability of D648 mannitol-copper oxide nanocomposites as phase change materials, J. Energy Storage 15 (2018) $649245-255$.

650 [12] V. Pethurajan, S. Sivan, A. Johny Konatt, A. Sarath Reddy, Facile approach to improve 651 solar thermal energy storage efficiency using encapsulated sugar alcohol based phase change 652 material, Sol. Energy Mater. Sol. Cells 185 (2018) 524-535.

653 [13] P. Ayazi Shamlow, A.G. Jones, K. Djamarani, Hydrodynamics of secondary nucleation in 654 suspension crystallization, Chem. Eng. Sci. 45 (5) (1990) 1405-1416.

655 [14] E. Aamir, Z.K. Nagy, C.D. Rielly, Evaluation of the effect of seed preparation method on 656 the product crystal size distribution for batch cooling crystallization processes, Crys. Growth 657 Des. 10 (11) (2010) 4728-4740.

658 [15] N.A. Clontz, W.L. McCabe, Contact nucleation of magnesium sulphate heptadydrate, 659 Chem. Eng. Symp. Ser. 110 (67) (1971) 6-17. 
[16] S.G. Agrawal, A.H.J. Paterson, Secondary nucleation: Mechanisms and models, Chem. Eng. Commun. 202 (5) (2015) 698-706.

662 [17] V. De Graef, B. Goderis, P. Van Puyvelde, I. Foubert, K. Dewettinck, Development of a rheological method to characterize palm oil crystallizing under shear, Eur. J. Lipid Sci. Technol.

$664110(2008) 521-529$.

665 [18] E. Tarabukina, F. Jego, J.M. Haudin, P. Navard, E. Peuvrel-Disdier, Effect of shear on the rheology and crystallization of palm oil, J. Food Sci. 74 (8) (2009) E405-E416 [19] M.L. Wolfrom, E.J. Kohn, Crystalline xylitol, J. Am. Chem. Soc. 64 (7) (1942) 1739-1739. [20] O. Seyvet, P. Navard. Collision-induced dispersion of agglomerate suspensions in a shear flow, J. Appl. Polym. Sci. 78 (5) (2000) 1130-1133.

670 [21] V. Collin, E. Peuvrel-Disdier, Dispersion mechanisms of carbon black in a elastomer 671 matrix, Elastomery 9 (2005) 9-15.

672 [22] M. Delgado, A. Lázaro, M. Biedenbach, S. Gamisch, S. Gschwander, S. Höhlein, A.

673 König-Haagen, D. Brüggemann, Intercomparative tests on viscosity measurements of phase

674 change materials, Thermochim. Acta 668 (2018) 159-168.

675 [23] M. Delgado, A. Lázaro, J. Mazo, C. Peñalosa, J.M. Marín, B. Zalba, Experimental analysis 676 of a coiled stirred tank containing a low cost PCM emulsion as a thermal energy storage system, 677 Energy 138 (2017) 590-601.

678 [24] J. Wu, L.J. Graham, N. Noui-Mehidi, Estimation of agitator flow shear rate, AIChE J. 52 679 (7) (2006) 2323-2332.

680 [25] A. Lázaro, C. Peñalosa, A. Solé, G. Diarce, T. Haussmann, M. Fois, B. Zalba, S.

681 Gschwander, L.F. Cabeza, Intercomparative tests on phase change materials characterisation 682 with differential scanning calorimeter, Appl. Energy 109 (2013) 415-420.

683 [26] G. Diarce, Development of new eutectic phase change materials and plate-based latent heat 684 thermal energy storage systems for domestic cogeneration applicatios. PhD Thesis. University 685 of the Basque Country (2017).

686 [27] L. Gora, R.W. Thompson, Investigations of secondary nucleation by initial breeding in 687 clear solution zeolite NaA systems, Zeolites 15 (6) (1995) 526-534

688 [28] H. Zhang. On sugar alcohol based heat storage materials: a nanoscale study and beyond. 689 PhD Thesis. Eindhoven University of Technology (2017).

690 [29] V. Collin, I. Boudimbou, E. Peuvrel-Disdier, New insights in dispersion mechanisms of 691 carbon black in a polymer matrix under shear by rheo-optics, J. Appl. Polym. Sci. 127 (3) 692 (2013) 2121-2131.

693 [30] D. Rusu, E. Peuvrel-Disdier, In-situ characterization by small angle light scattering of the 694 shear-induced coalescence mechanism in inmiscible polymer blends, J. Rheol. 43 (6) (1999) 695 1391-1409.

696 [31] S.A.E. Boyer, J.M. Haudin, Crystallization of Polymers in Processing Conditions: An 697 Overview, Intern. Polym. Proc. 32 (5) (2017) 545-554.

698 [32] E. Palomo del Barrio, A. Godin, M. Duquesne, J. Daranlot, J. Jolly, W. Alshaer, T. 699 Kouadio, A. Sommier, Characterization of different sugar alcohols as phase change materials 700 for thermal energy storage applications, Sol. Energy Mater. Sol. Cells 159 (2017) 560-569. 
701 [33] I.M. Krieger, T.J. Dougherty, A mechanism for non-Newtonian flow in suspensions of 702 rigid spheres, T. Soc. Rheol. 3 (1959) 137-152.

703 [34] R.G. Larson, The structure and rheology of complex fluid, in: K.E. Gubbins editor, Oxford 704 Univ. Press, New York, 1999.

705 [35] Y.S. Papir, I.M. Krieger, Rheological studies on dispersions of uniform coloidal spheres-II. 706 Dispersions in non-aqueous media, J. Colloid. Int. Sci. 34 (1970) 126-130.

707 [36] J.Z.Q. Zhou, T. Fang, G. Luo, P.H.T. Uhlherr, Yield stress and maximum packing fraction 708 of concentrated suspensions, Rheol. Acta 34 (1995) 544-561.

709 [37] E. Palomäki, P. Ahvenainen, H. Ehlers, K. Svedström, S. Huotari, J. Yliruusi, Monitoring 710 the recrystallization of amorphous xylitol using Raman spectroscopy and wide-angle X-ray 711 scattering, Int. J. Pharm. 508 (1-2) (2016) 71-82.

712 [38] J.D. Dunitz, J. Bernstein, Disappearing polymorphs, Acc. Chem. Res. 28 (1995) 193-200. 713

\section{Appendix A.}

715

\begin{tabular}{|c|c|c|c|c|c|c|}
\hline & \multirow[b]{2}{*}{ Measurement } & \\
\hline & & $F_{n 0}$ & $\mathbf{A}$ & $\mathbf{w}$ & $\mathbf{t}_{\mathrm{c}}$ & $\mathbf{R}^{2}$ \\
\hline \multirow{3}{*}{$5 s^{-1}$} & 1 & -0.02574 & 32176.71989 & 327.24623 & 789 & 0.99499 \\
\hline & 2 & 1.55047 & 27403.15938 & 424.3942 & 1206 & 0.98359 \\
\hline & 3 & -0.02822 & 21331.48656 & 312.03773 & 617 & 0.99683 \\
\hline \multirow{3}{*}{$10 \mathrm{~s}^{-1}$} & 1 & -0.18121 & 17416.45673 & 242.09359 & 504 & 0.98830 \\
\hline & 2 & 0.60613 & 8082.09301 & 153.43878 & 376 & 0.99772 \\
\hline & 3 & -1.69251 & 22316.93972 & 294.69705 & 475 & 0.96708 \\
\hline \multirow{3}{*}{$15 \mathrm{~s}^{-1}$} & 1 & 0.16724 & 11267.1018 & 165.26012 & 479 & 0.99944 \\
\hline & 2 & 0.11948 & 22489.49646 & 318.98937 & 1097 & 0.97434 \\
\hline & 3 & 0.45776 & 25486.66224 & 562.76256 & 1553 & 0.99797 \\
\hline
\end{tabular}



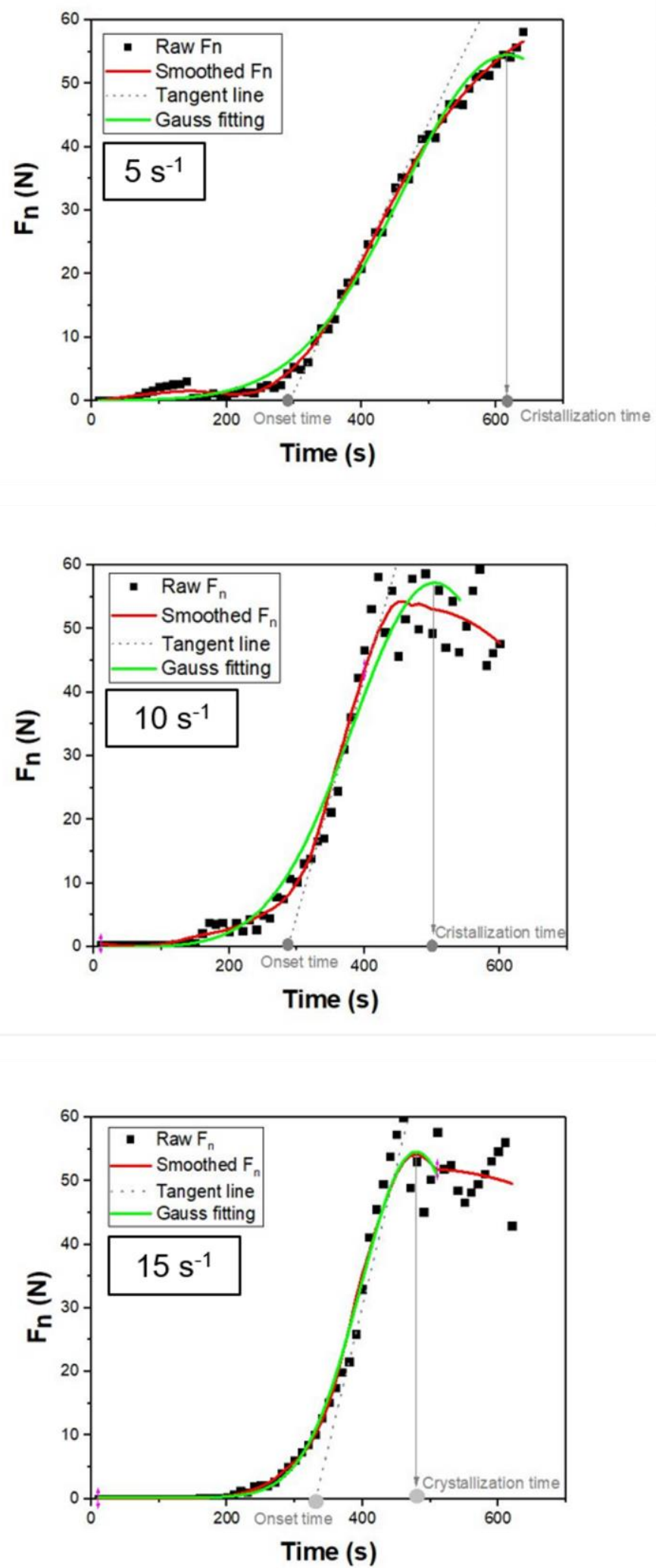

Figure A.1. Procedure to obtain the onset and crystallization time from the normal force evolution at 5,10 and $15 \mathrm{~s}^{-1}$ 